\title{
ETIOLOGÍA Y EPIDEMIOLOGÍA DE LA NECROSIS FLORAL DEL ZAPOTE MAMEY (Pouteria sapota (Jacq.) H.E. Moore y Stearn) EN GUERRERO, MÉXICO
}

\author{
ETIOLOGY AND EPIDEMIOLOGY OF FLORAL NECROSIS IN SAPOTE MAMEY \\ (Pouteria sapota (Jacq.) H.E. Moore y Stearn) IN GUERRERO, MÉXICO
}

\author{
Alfonso Vásquez-López ${ }^{1,2 \star}, J^{\text {. Antonio Mora-Aguilera }}{ }^{1}$, Cristian Nava-Díaz ${ }^{1}$ y Daniel Téliz-Ortiz ${ }^{1}$
}

\begin{abstract}
${ }^{1}$ Postgrado en Fitosanidad-Fitopatología, Colegio de Postgraduados-Campus Montecillo. Km. 36.5 carretera México-Texcoco. 56230, Montecillo, Texcoco, Edo. de México, México. ${ }^{2}$ Dirección Actual: Centro Interdisciplinario de Investigación para el Desarrollo Integral Regional-Unidad Oaxaca, Instituto Politécnico Nacional. Calle Hornos 1003. 71230, Santa Cruz Xoxocotlán, Oax. Tel y Fax 01 (951) 5170610 ext. 82705.
\end{abstract}

*Autor para correspondencia (avasquez@ipn.mx)

\section{RESUMEN}

En Alpoyeca, Guerrero, México, la necrosis floral del mamey (Pouteria sapota) es una enfermedad de etiología desconocida que se presenta con alta incidencia (80 a $100 \%)$ y reduce la productividad de los huertos comerciales. Los objetivos del presente estudio fueron determinar la etiología y el progreso temporal de la enfermedad. De flores necróticas se aisló a Penicillium olsonii y Alternaria alternata y de flores asintomáticas Pestalotiopsis paeoniicol. La patogenicidad de los hongos se verificó en flores asintomáticas en 2005 y 2006. P. olsonii y A. alternata, inoculados en suspensión conidial $\left(1 \times 10^{3}\right.$ conidios $\left.\mathrm{mL}^{-1}\right)$, causaron necrosis, marchitez, pudrición y abscisión floral a los 5 y $8 \mathrm{~d}$, respectivamente. $P$. paeoniicola indujo necrosis de pétalos después de $8 \mathrm{~d}$. El progreso temporal de la necrosis floral se estudió en 10 árboles de mamey de mayo a agosto de 2005; la incidencia final osciló entre 80 y $100 \%$. De las epidemias, 50 \% se ajustaron al modelo Gompertz, $30 \%$ al logístico y $20 \%$ al monomolecular. La incidencia de la enfermedad se asoció con humedad relativa de 65 a $75 \%(r=0.80)$ con la velocidad del viento $\leq 5 \mathrm{Km} \mathrm{h}^{-1}(r=0.75)$. La densidad conidial de Alternaria sp. y Pestalotiopsis sp. se relacionó con la humedad relativa de 70 a $90 \%$ $\left(r_{\text {Alternaria }}=0.75\right.$ y $\left.r_{\text {Pestalotiopsis }}=0.80\right)$ y con velocidad del viento $\leq 5 \mathrm{~km} \mathrm{~h}^{-1}$ para Alternaria sp. $\left(r_{\text {Alternaria }}=0.70\right)$. En Alpoyeca, Guerrero, la necrosis floral del mamey fue inducida por Penicillium olsonii y Alternaria alternata. La intensidad de la enfermedad se correlacionó con humedad relativa y velocidad del viento.

Palabras clave: Pouteria sapota, Penicillium olsonii, Alternaria alternata, Pestalotiopsis paeniicola, densidad conidial.

\section{SUMMARY}

In Alpoyeca, Guerrero, México, floral necrosis is a disease of unknown etiology that occurs with high incidence (80 to $100 \%$ ) in sapotes mamey (Pouteria sapota) and reduces the productivity of commercial orchards. The objectives of the present study were to determine the etiology and temporal progress of the disease. Penicillium olsonii and Alternaria alternata were isolated from necrotic flowers, and Pestalotiopsis paeoniicola was isolated from asymptomatic flowers. The pathogenicity of the fungi was verified in asymptomatic flowers in 2005 and 2006. P. olsonii and A. alternate, inoculated in a conidial suspension $\left(1 \times 103\right.$ conidia $\left.\mathrm{mL}^{-1}\right)$ caused necrosis, wilting, rot, and floral abscission at $5 \mathrm{~d}$ and $8 \mathrm{~d}$, respectively. $P$. paeoniicola induced petal necrosis after $8 \mathrm{~d}$. The temporal progress of floral necrosis was studied on 10 mamey sapote trees from May to August of 2005, with a final incidence varying between 80 and $100 \%$. Fifty percent of the epidemics fit the Gompertz model, $30 \%$ fit the logistic model, and $20 \%$ fit the monomolecular model. Disease incidence was associated with relative humidity of 65 to $75 \%(r=0.80)$ and greater than $5 \mathrm{~km}$ $\mathbf{h}^{-1}$ wind speed $(r=0.75)$. The conidial densities of Alternaria sp. and Pestalotiopsis sp. were correlated with relative humidity from 70 a $90 \%$ $\left(r_{\text {Alternaria }}=0.75\right.$ and $\left.r_{\text {Pestalotiopsis }}=0.80\right)$ and with greater than $5 \mathrm{~km} \mathrm{~h}^{-1}$ wind speed for Alternaria sp. $\left(r_{\text {Alternaria }}=0.70\right.$. In Alpoyeca, Guerrero, floral necrosis of mamey was induced by Penicillium olsonii and Alternaria alternata. The intensity of the disease was correlated with relative humidity and wind speed.

Index words: Pouteria sapota, Penicillium olsonii, Alternaria alternata, Pestalotiopsis paeoniicola, conidial density.

\section{INTRODUCCIÓN}

El mamey (Pouteria sapota (Jacq.) H.E. Moore y Stearn) se cultiva en los países que conforman el centro de origen y diversidad de la especie, entre ellos México, Guatemala, El Salvador, Honduras, Costa Rica, Cuba y EE. UU. (Azurdia, 2006). En México, en el año 2011 se cultivaron 1702 ha con una producción estimada de 16357 t. Las entidades federativas con mayor superficie y producción fueron Yucatán (492 ha y 9448 t), Guerrero (390 ha y 2270 t) y Michoacán (234 ha y 673 t) (SIAP, 2011). En Huamuxtitlán y Alpoyeca, municipios situados en la montaña baja del Estado de Guerrero, se cultivan aproximadamente 250 ha con mamey con alto potencial de producción y alta rentabilidad, cuya producción total de fruto fresco es consumida por el mercado nacional.

En esta región la necrosis floral del mamey es una enfermedad de etiología desconocida que disminuye hasta en 35 $\%$ la productividad de los huertos. La enfermedad se detecta en flores recién abiertas cuyos pétalos muestran micelio de color verde-olivo con esporulación abundante (Figura 1A), micelio de color café oscuro con esporulación escasa (Figura 1B) o desarrollo de acérvulos (Figura 1C). 
Estos organismos fungosos provocan lesiones necróticas y marchitez de la corola; posteriormente colonizan todos los órganos florales e inducen necrosis, marchitez y abscisión. No se encontraron antecedentes sobre el origen de la enfermedad; sólo se había documentado que las flores pueden sufrir antracnosis inducida por Colletotrichum gloeosporioides (Penz.) Penz. \& Sacc. (Morton, 1987; Balerdi et al., 2005). Tampoco hay registros sobre el comportamiento epidémico de la necrosis floral.

Conocer el origen y progreso de una enfermedad permite identificar fases epidémicas críticas como procesos de dispersión, periodos de mayor infección, y condiciones climáticas que promueven el desarrollo del parásito y la enfermedad. Estudios de esta naturaleza son necesarios para desarrollar un manejo fitosanitario sustentable y efectivo de la necrosis floral del mamey. Por ello, el presente estudio tuvo como objetivo determinar la etiología y el progreso temporal de la enfermedad necrosis floral de mamey.

\section{MATERIALES Y MÉTODOS}

\section{Sitio de estudio y aislamiento de hongos}

En Alpoyeca, Guerrero, México ( $17^{\circ} 40^{\prime} \mathrm{LN}$ y $98^{\circ} 31^{\prime}$ LO, $960 \mathrm{~m}$ ), en el año 2005 se seleccionaron 30 árboles de mamey de 20 años de edad con flores necróticas y de cada uno se colectaron dos flores con necrosis, una de ellas con estructuras fungosas color verde-olivo y la otra con signos fungosos color café-oscuro; en total se recolectaron 60 flores enfermas. De cada flor se cortaron dos secciones de pétalos de $0.5 \mathrm{~cm}^{2}$, se lavaron con agua, se desinfestaron con hipoclorito de sodio a $1.0 \%$ por $3 \mathrm{~min}$, se lavaron tres veces con agua destilada estéril, se secaron con toallas de papel y se sembraron en medio de cultivo papa-dextrosa-agar (PDA) (BD Bioxon ${ }^{\circledR}$, Becton Dickinson de México). Las cajas de Petri estuvieron incubadas a $25^{\circ} \mathrm{C}$ con luz blanca por $3 \mathrm{~d}$; las colonias fungosas que se desarrollaron se transfirieron individualmente a PDA, se incubaron a $25^{\circ} \mathrm{C}$ con luz blanca por $10 \mathrm{~d}$, y se purificaron por cultivos monoconidiales.

\section{Pruebas de patogenicidad}

La patogenicidad de los hongos aislados se verificó en flores asintomáticas de mamey en el verano del 2005 y el invierno del 2006. Cada hongo inoculado fue un tratamiento, más un testigo; la unidad experimental fue una flor. El inóculo se preparó mediante cultivo individual de los hongos en PDA, a $25{ }^{\circ} \mathrm{C}$ con luz blanca por $10 \mathrm{~d}$. De cada hongo se preparó una solución conidial concentrada a $1 \mathrm{x}$ $10^{3}$ conidios $\mathrm{mL}^{-1}$. Previo a la inoculación, se seleccionaron 60 flores experimentales para cada tratamiento las cuales permanecieron adheridas al árbol durante el estudio. Las flores de cada tratamiento se desinfestaron con hipoclorito de sodio a $0.5 \%$ por $2 \mathrm{~min}$, se enjuagaron tres veces con
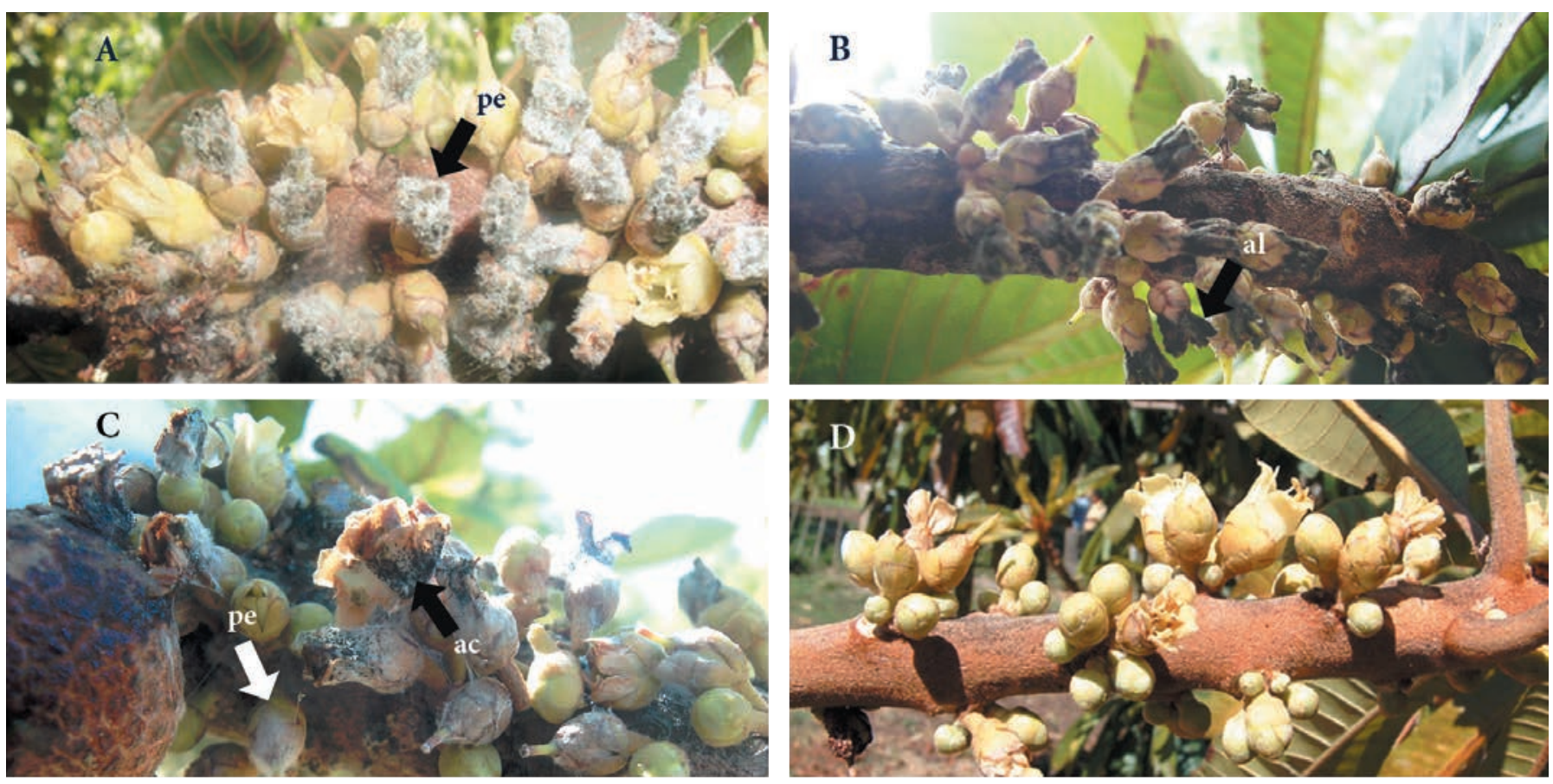

Figura 1. Flores de mamey (P. sapota) con infección fungosa natural (infección en campo). A) Flores infectadas con Penicillium sp. (pe). B) Flores infectadas con Alternaria sp. (al). C) Flores con signos de Pestalotiopsis sp. (ac) y Penicillium sp. (pe). D) Flores asintomáticas. 
agua destilada estéril y se asperjaron con $3 \mathrm{~mL}$ de solución conidial. El testigo se asperjó con agua destilada estéril. Las flores inoculadas se cubrieron durante los primeros $5 \mathrm{~d}$ con una bolsa de plástico desinfestada con alcohol y dentro se colocó un algodón estéril humedecido con agua destilada estéril. La incidencia de la necrosis floral por tratamiento se estimó con la ecuación: $I i=(\Sigma n i / N i)$, donde: $I i=$ incidencia de flores necróticas en el momento $i$; $n i=$ número de flores necróticas en el momento $i ; \mathrm{Ni}=$ población total de flores inoculadas. La evaluación se realizó a 3, 5, 8 y 12 $\mathrm{d}$ después de la inoculación (ddi). De tejidos infectados se reaislaron a los hongos en cultivo puro y se compararon morfológicamente con los aislamientos originales.

\section{Caracterización morfológica y molecular de fitopatógenos}

Los hongos patógenos se identificaron con la clave taxonómica para género de Barnett y Hunter (2006). La especie de Penicillium se determinó con las referencias de Raper y Thom (1949) y Samson et al. (2004); la especie de Pestalotiopsis con las claves de Guba (1961) y Wei et al. (2005); y la especie de Alternaria con la clave de Rotem (1994).

El ADN de los hongos fitopatógenos se extrajo con la técnica de Ahrens y Seemüller (1992). La amplificación de las regiones ITS1 e ITS2 de los genes ribosomales se hizo por PCR con los iniciadores ITS4 (TCC TCC GCT TAT TGA TAT GC) e ITS5 (GGA AGT AAA AGT CGT AAC AAG G) (White et al., 1990), con la siguiente modificación: agua ultrapura estéril $(13.22 \mu \mathrm{L})$, solución amortiguadora TBE $1 \mathrm{X}(2.5 \mu \mathrm{L}), \mathrm{MgCl}_{2}$ a $2.5 \mathrm{mM}(2.08 \mu \mathrm{L})$, dNTPs a $0.2 \mathrm{mM}(2$ $\mu \mathrm{L})$, iniciadores ITS4 e ITS5 a $20 \rho \mathrm{mol}(2 \mu \mathrm{L}$ de cada uno), DNA polimerasa (Biogenica $\left.{ }^{\circledR}\right)$ a $1 \mathrm{U}(0.2 \mu \mathrm{L})$, y muestra problema de $\mathrm{ADN}$ a $80 \mathrm{ng}(1 \mu \mathrm{L})$. El producto amplificado se purificó con el juego de Wizard (Promega ${ }^{\circledR}$ ) y se secuenció con el analizador genético modelo 3100 de Applied Biosystem ${ }^{\circledR}$. Las secuencias se analizaron con el programa Lasergene ${ }^{\circledR} 2001$, V5 (DNASTAR, Inc.) y se alinearon con la base de datos del banco de genes del National Center for Biotechnology Information (NCBI) (www.ncbi.nlm.nih. gov). Las secuencias con el valor más alto de identidad se compararon con las de este estudio.

\section{Incidencia de la necrosis floral y análisis de epidemias}

El progreso temporal de la enfermedad se estudió en 10 árboles de mamey de tipo criollo de 25 años de edad. En un huerto (HE1), sin manejo agronómico, se eligieron cinco árboles de $25 \mathrm{~m}$ de altura y copas entrelazadas. En otro huerto (HE2), con manejo agronómico (podas, fertilización, riego), se seleccionaron cinco árboles de $10 \mathrm{~m}$ de altura sin contacto foliar entre ellos. De cada árbol se eligieron
400 botones florales en los que se estimó la incidencia de la enfermedad de mayo a agosto de 2005, en intervalos de 15 d, mediante la ecuación citada en la sección "pruebas de patogenicidad". La intensidad de la enfermedad por árbol se calculó con el área bajo la curva del progreso de la enfermedad (abcpe) obtenida por el método de integración trapezoidal (Campbell y Maden, 1990).

El modelo epidemiológico para cada árbol experimental se obtuvo con los datos porcentuales de incidencia estandarizados por el método de Haldane (1956), y se transformaron con: logaritmo (incidencia), log (1/(1-incidencia), $\log$ (incidencia/(1-incidencia)) y - $\log (-\log$ (incidencia)). La incidencia a través del tiempo se ajustó con los modelos matemáticos exponencial, monomolecular, logístico y Gompertz (Campbell y Madden, 1990). El modelo elegido para cada epidemia fue el que dio el mayor coeficiente de determinación $\left(\mathrm{R}^{2}\right)$.

Para comparar las epidemias fue necesario homologar los modelos exponencial, monomolecular y logístico con el modelo Gompertz. La homologación se hizo con los parámetros rho y $k_{G}$ estimados de los tres primeros modelos: $r h o=k /((m \times 2)+2)$ (Richards, 1959), donde $r h o=$ tasa de homologación entre epidemias; $k=$ tasa de incremento de la enfermedad del modelo a homologar; $m$ adquiere valores de 0,1 y 2 para los modelos monomolecular, Gompertz y logístico, respectivamente; $k_{G}=$ rho $\times((m \times 2)+2)$, donde $k_{G}=$ tasas homologadas al modelo de Gompertz; $m=1$, debido a que las tasas de incremento se homologan al modelo Gompertz.

\section{Estudios de correlación}

Se analizó la relación del progreso temporal de la enfermedad con temperatura $\left({ }^{\circ} \mathrm{C}\right)$, humedad relativa (\%) y velocidad del viento $\left(\mathrm{km} \mathrm{h}^{-1}\right)$, de mayo a agosto de 2005 , mediante el coeficiente de correlación de Pearson ( $r$ ); también se relacionó la densidad conidial de Alternaria sp. y Pestalotiopsis sp. de enero a diciembre de 2007, con los siguientes intervalos de las variables climáticas: temperatura ( $\leq 19.9,20-25,25.1-30,30.1-35, \geq 35.1)$, humedad relativa $(\leq 50,51-60,61-70,71-80,81-90, \geq 91)$ y velocidad del viento $(\leq 2,2.1-5, \geq 5.1)$.

\section{Variables climáticas y monitoreo de esporas}

Las variables climáticas se registraron con una estación meteorológica Vantage Pro ${ }^{\circledR}$ modelo 6310 (Davis Instruments) en intervalos de $2 \mathrm{~h}$, de mayo a agosto de 2005. La consola se automatizó con el programa WeatherLink ${ }^{\circledR}$ versión 5.4 para Windows (Davis Instruments). La densidad diaria de esporas en el aire de Alternaria spp. y Pestalotiopsis spp. se cuantificó de enero a diciembre de 
2007 con una trampa volumétrica tipo Burkard (Gadoury y MacHardy, 1983), modificada por Mora (2000) (Com. personal $^{1}$ ). Las esporas capturadas por impacto sobre una cinta adhesiva transparente de polipropileno (Contac ${ }^{\circledR}$ ) se cuantificaron con microscopía de luz.

\section{RESULTADOS Y DISCUSIÓN}

\section{Hongos aislados}

De 60 secciones de pétalos necróticos con signos fungosos color verde-olivo se aisló a Penicillium sp. (100\%) y a Pestalotiopsis sp. (15\%). De 60 secciones de pétalos necróticos con micelio café-oscuro se aisló a Alternaria sp. (100\%), Penicillium sp. (30 \%) y Pestalotiopsis sp. (10\%). De pétalos asintomáticos se aisló a Pestalotiopsis sp. (80 \%).

\section{Pruebas de patogenicidad}

Las flores inoculadas con Penicillium sp. desarrollaron signos fungosos de color verde-olivo. A 3 ddi el hongo colonizó pétalos, estambres, ovario y sépalos en 60 y $52 \%$ de las flores experimentales en verano e invierno (V/I); a 5 ddi se detectaron lesiones necróticas y marchitez de corola en 80 y $75 \%$ de las flores de V/I; y a 8 ddi la incidencia de necrosis y abscisión floral final fue de 95 y $92 \%$ (Figura 2A). Las flores inoculadas con Alternaria sp. desarrollaron micelio de color café oscuro; este hongo colonizó pétalos, estambres, ovario y sépalos en 45 y $42 \%$ de las flores experimentales de V/I, a 5 ddi; a 8 ddi causó marchitez y necrosis floral con abundante desarrollo micelial (Figura 2B) y a 12 ddi la incidencia de pudrición y abscisión floral fue de 85 y 76 \%. Pestalotiopsis sp. produjo acérvulos y necrosó la corola en 55 y $38 \%$ de las flores de V/I, a 5 ddi; a 8 ddi, 70 y $65 \%$ de las flores de V/I presentaron necrosis y caída de pétalos, con pudrición de cáliz y pedúnculo (Figura $2 \mathrm{C}$ ). En las flores testigo, $53.3 \%$ de verano y $33.3 \%$ de invierno, se desarrollaron acérvulos de Pestalotiopsis a 8 ddi (Figura 2D), lo cual evidenció una infección preexistente del hongo debido a su hábito endófito en los árboles de mamey (Gómez et al., 2009). Los síntomas reproducidos en flores inoculadas fueron similares a los que se presentaron por infección natural en Alpoyeca, Gro.

\section{Caracterización morfológica y molecular de fitopatógenos}

Penicillium olsonii Bainier y Sartory. La colonia de $10 \mathrm{~d}$ de edad crecida en medio de cultivo con extracto de malta (MEA) exhibió color verde olivo con micelio aéreo, algodonoso y septado. Conidióforos largos y rectos, penicilo ter-

${ }^{1}$ Mora A A (2000) Patogénesis y epidemiología de la "escoba de bruja" (Fusarium subglutinans (Wollenweb. \& Reinking) y F. oxysporum (Schlecht)) del mango (Mangifera indica L.) en Michoacán, México. Tesis Doctoral. Colegio de Postgraduados. Texcoco, México. 107 p. verticilado y compacto con métulas de 9-12 x 3.3-5.5 $\mu \mathrm{m}$. Fiálides en forma de botella con cuello corto de 9.3-9.8 x 2.3-2.6 $\mu \mathrm{m}$. Amerosporas ovoides con pared rugosa de 3.44 x 2.4-2.9 $\mu \mathrm{m}$ (Figuras 3A-3D). Las características morfométricas de este aislamiento coinciden con las reportadas por Raper y Thom (1949) y Samson et al. (2004).

Alternaria alternata (Fr.) Keissler. La colonia en PDA a 15 d de incubación exhibió color café oscuro con micelio aéreo y septado. Conidióforos erectos e individuales de 28.9-49 x 3-3.3 $\mu \mathrm{m}$. Dictiosporas obpiriformes o elipsoides de 22.3-35.7 x 7.3-12.6 $\mu \mathrm{m}$ con longitud apical de 6.6-8.9 $\mu \mathrm{m}$; 3-5 transeptos, 1-2 longiseptos, catenulados (6 a 10 conidios por cadena). Las características de este aislamiento concuerdan con las reportadas por Rotem (1994).

Pestalotiopsis paeoniicola (Tsukamoto y Hino) J. G. Wei y T. Xu. La colonia en PDA a 15 d de incubación exhibió color blanco con micelio aéreo, algodonoso y septado. Esporodoquios negros esparcidos en el medio de cultivo. Fragmosporas de cinco células, fusiformes o alantoides (rectos o ligeramente curvos) de 23.09-26.73 x 6.08-7.29 $\mu \mathrm{m}$ (promedio $25 \times 6.91 \mu \mathrm{m}$ ); tres células intermedias de color café de $14.58 \times 17.25 \mu \mathrm{m}$ (promedio $16.25 \mu \mathrm{m}$ ); célula apical hialina de forma cónica con dos o tres apéndices hialinos, sin ramificaciones, de 18.22 a $27.95 \mu \mathrm{m}$ de longitud (promedio $22.20 \mu \mathrm{m}$ ). Célula basal hialina de forma cónica con un solo apéndice central hialino y sin ramificaciones (Figuras 4A-4C). Relación largo/ancho de los conidios 3.62:1. Las características de este aislamiento coinciden con las reportadas por Guba (1961), Wei et al. (2005) y Gómez et al. (2009).

Las secuencias experimentales tuvieron 98, 95 y $97 \%$ de identidad con los aislamientos de Penicillium olsonii (DQ123662), Pestalotiopsis paeoniicola (AY687310) y Alternaria alternata (AY714479) depositados en el banco de genes (NCBI, 2008). Antes de este estudio no se encontraron reportes de $P$. olsonii, A. alternata o P. paeniicola como patógenos de flores de mamey; sólo se había documentado que las flores sufrían antracnosis por el ataque de C. gloeosporioides en Florida, EE. UU. (Balerdi et al., 2005; Mossler y Crane, 2009); sin embargo la enfermedad no se consideró de importancia económica.

\section{Incidencia de la necrosis floral}

La incidencia inicial $\left(Y_{o}\right)$ de la enfermedad en flores de HE1 se presentó a partir de la cuarta semana de mayo del 2005 y se incrementó en los primeros 15 d hasta en 80 $\%$. La incidencia final $\left(Y_{f}\right)$ fue de $100 \%$ a principios de agosto (Figura 5A). Mientras que la $Y_{o}$ en flores de HE2 se presentó hasta la cuarta semana de junio del 2005, y en los primeros $15 \mathrm{~d}$ la incidencia se incrementó hasta en $64 \%$. 

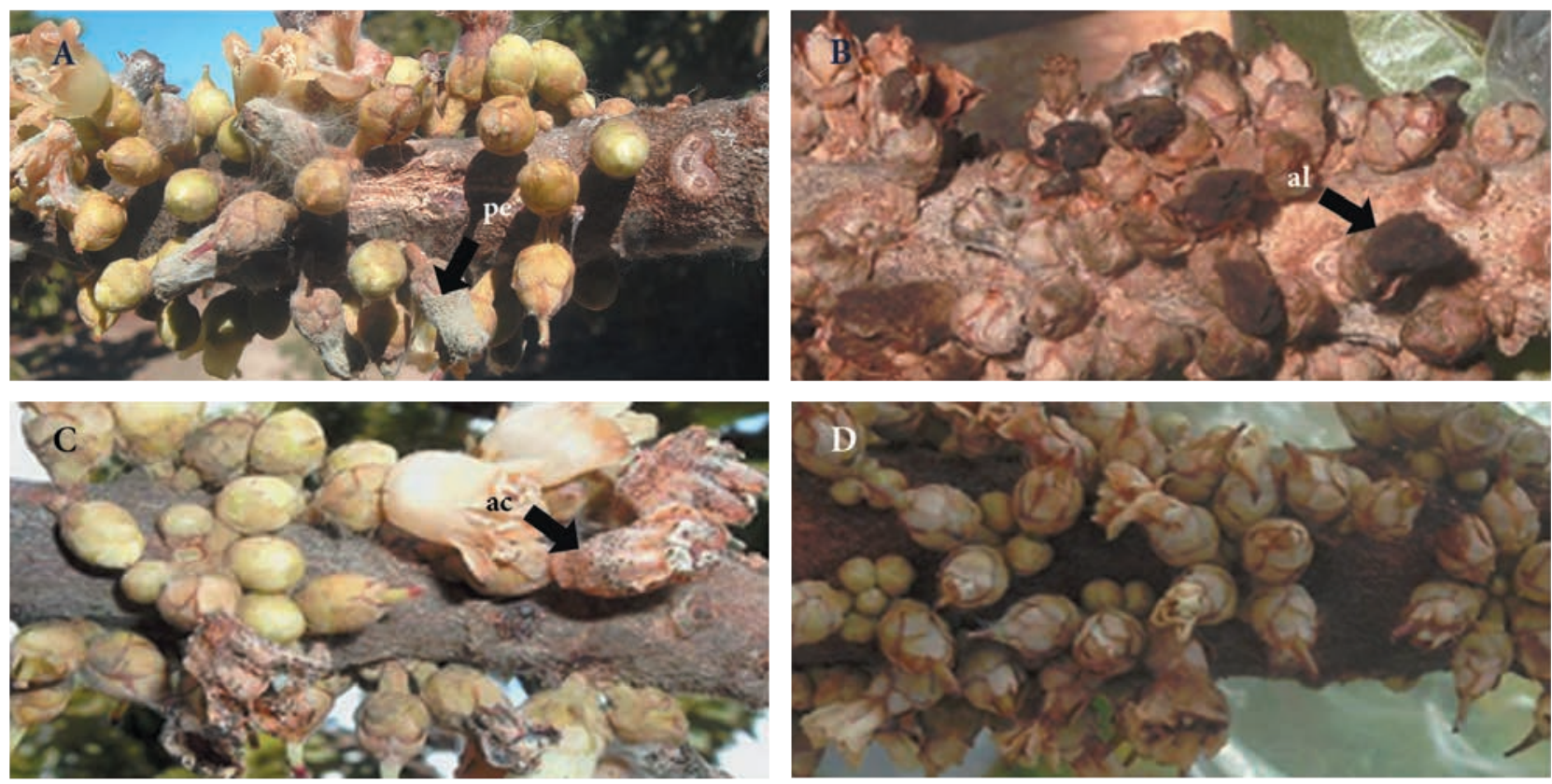

Figura 2. Verificación patogénica de hongos aislados de flores enfermas de mamey. A) Penicillium sp. (pe) en flores experimentales, a 3 días de la inoculación (ddi). B) Necrosis y marchitez floral inducida por Alternaria sp. (al), a 8 ddi. C) Presencia de acérvulos (puntos negros) y necrosis en flores inoculadas con Pestalotiopsis sp. (ac), a 8 ddi. D) Flores testigo.

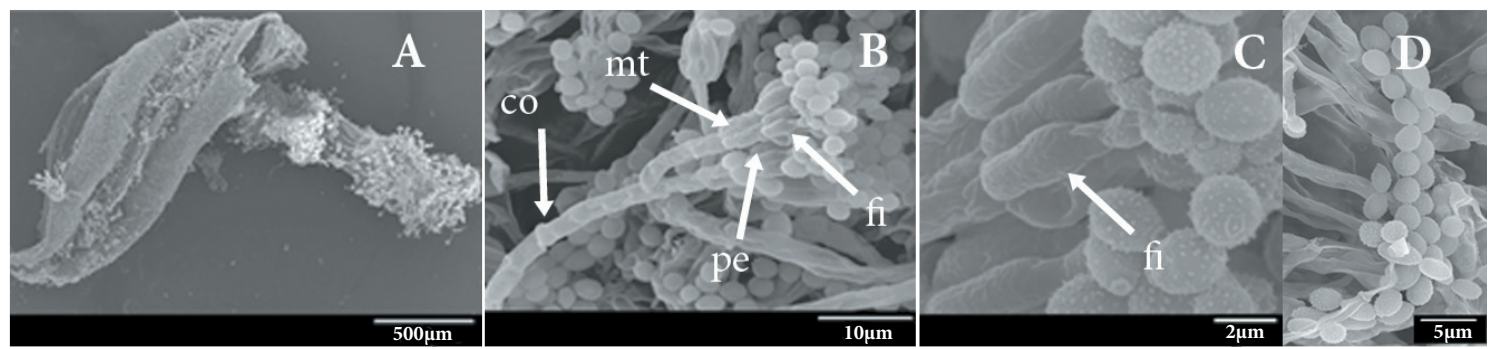

Figura 3. Penicillium olsonii. A) Signos fungosos en el estambre de una flor de mamey, a 8 ddi. B) Conidióforos (co) con penicilo (pe) terverticilado, penicilo con métulas (mt) y fiálides (fi). C) Fiálides rectas con forma de botella (fi). D) Conidios amerosporas en cadena, ovoides y de pared rugosa. Fotomicrografías en microscopio de barrido JSM-6390/LGS. 2007.
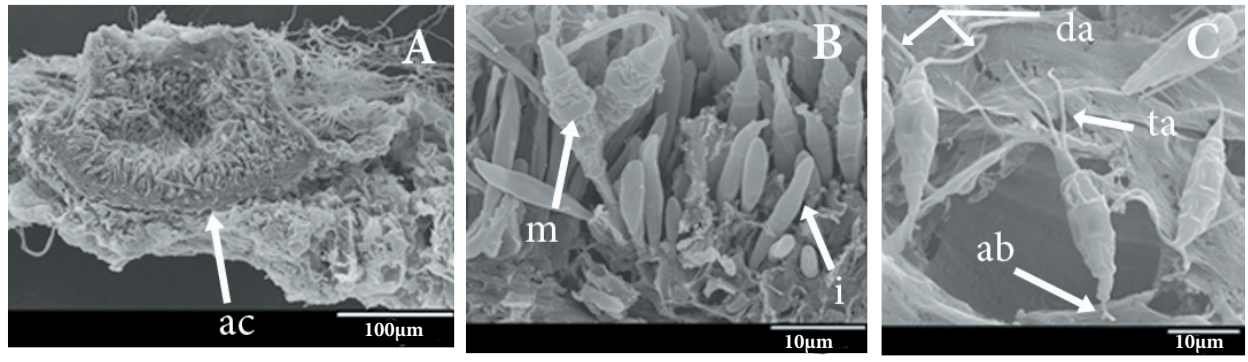

Figura 4. Pestalotiopsis paeoniicola. A) Acérvulo subepidérmico (ac) en un pétalo, a 8 ddi. B) Conidios maduros (m) e inmaduros (i) dentro de un acérvulo. C) Conidios (fragmosporas) fusiformes o alantoides, con dos (da) y tres (ta) apéndices apicales y un apéndice basal (ab). Fotomicrografías en microscopio de barrido JSM-6390/LGS. 
En promedio, $Y_{f}$ fue de $80 \%$ en la tercera semana de agosto (Figura 5B). En el periodo de evaluación la temperatura varió de 22 a $25^{\circ} \mathrm{C}$ y la $\mathrm{HR}$ de 70 a $90 \%$.

La intensidad de enfermedad fue mayor en los árboles experimentales de HE1 respecto a los árboles de HE2. En HE1 la cantidad de enfermedad fluctúo de 3278 a 4514 unidades (abcpe) (Figura 5A) y en HE2 fluctuó de 2225 a 3618 unidades (Figura 5B). El comportamiento de las epidemias en ambos huertos puede explicarse por las condiciones contrastantes de manejo agronómico del cultivo. En HE1 los árboles experimentales fueron de $25 \mathrm{~m}$ de altura sin podas de saneamiento, con contacto entre ramas de árboles vecinos que restringe la circulación del aire y probablemente genera un microclima en el que la HR favorece el rápido crecimiento de los hongos. Las ramas reproductivas de los árboles en HE1 permanecieron mojadas por más tiempo en verano y tuvieron agua libre (condensada) por más tiempo que en HE2. En contraste, los árboles en HE2 de $10 \mathrm{~m}$ de altura no tuvieron contacto entre ramas de árboles vecinos, y posiblemente la mejor ventilación retrasó el crecimiento de los hongos.

\section{Análisis de epidemias}

En HE1, 60 \% de las epidemias se ajustaron al modelo Gompertz $\left(\mathrm{R}^{2}=0.93,0.92\right.$ y 0.78$), 20 \%$ al monomolecular $\left(\mathrm{R}^{2}=0.99\right)$ y $20 \%$ al logístico $\left(\mathrm{R}^{2}=0.70\right)$ (Cuadro 1$)$. En HE2, $40 \%$ de las epidemias fueron descritas por el modelo Gompertz $\left(\mathrm{R}^{2}=0.98\right.$ y 0.87$)$, igual porcentaje se ajustó al logístico $\left(R^{2}=0.94\right.$ y 0.96$)$, y $20 \%$ fue descrito por el monomolecular $\left(\mathrm{R}^{2}=0.75\right)$. El modelo epidemiológico para cada árbol experimental, así como los parámetros de homologación ( $r h o$ y $k_{G}$ ) de los modelos monomolecular y logístico, se muestran en el Cuadro 1.

\section{Estudios de correlación}

La incidencia de la necrosis floral se relacionó con la humedad relativa de 65 a $75 \%(r=0.80)$ y con la velocidad del viento $\leq 5 \mathrm{~km} \mathrm{~h}^{-1}(r=0.75)$. La densidad conidial en el aire de Alternaria sp. y Pestalotiopsis sp. se asoció con la humedad relativa de 70 a $90 \%\left(r_{\text {Alternaria }}=0.75\right.$ y $\left.r_{\text {Pestalotiopsis }}=0.80\right)$, y con la velocidad del viento $\leq 5 \mathrm{~km} \mathrm{~h}^{-1}$ para Alternaria sp. $\left(r_{\text {Alternaria }}=0.70\right)$. La temperatura de 20 a $25^{\circ} \mathrm{C}$ favoreció a Pestalotiopsis sp. $\left(r_{\text {Pestalotiopsis }}=0.65\right)$.

Los factores ambientales considerados fueron útiles para el estudio epidémico de la necrosis floral del mamey, porque permitieron conocer los intervalos que favorecen su desarrollo. Con un enfoque similar, Mora (2000; Com. Personal) estudió la epidemiología de la "escoba de bruja" (Fu- sarium oxysporum y F. subglutinans) del mango (Mangifera indica) en Michoacán, y documentó que la enfermedad se correlacionó con el número de esporas de Fusarium spp., y el inóculo en el aire correlacionó con temperatura, humedad relativa, velocidad y dirección del viento. Según Rotem (1994) y Timmer et al. (1998), los principales factores que intervienen en la liberación de esporas de varias especies de hongos, entre ellos Alternaria spp., son humedad relativa, temperatura, luz roja e infrarroja, humedad de la hoja, velocidad del viento, vibración de la hoja y los periodos de lluvia.

Conocer los factores ambientales que favorecen la esporulación y liberación de esporas ayuda a tomar medidas profilácticas oportunas (aspersión de fungicidas, podas de ventilación, etc.) para proteger temporalmente las etapas más susceptibles de las plantas, al reducir la liberación de esporas (Gottwald et al., 1997) y limitar el número de infecciones potenciales. En el huerto de mamey de Alpoyeca, Gro. se capturaron esporas de Alternaria spp. y Pestalotiopsis spp. en la mayor parte del año, aunque la captura fue mayor durante la floración de verano que ocurre de junio a noviembre, por lo que esta etapa fenológica (floración) representa un sustrato altamente favorable para incrementar las poblaciones del hongo (presión de infección), y a la vez identifica el periodo para ejercer un control químico oportuno y racional.

Con base en los modelos que describen el progreso de la enfermedad a través del tiempo, se puede afirmar que estos tres patógenos tienen un comportamiento policíclico, por lo que aplicaciones previas durante la etapa fenológica susceptible y en las condiciones ambientales favorables para los hongos, tienen el potencial de reducir el impacto de la enfermedad en campo. No se encontraron estudios publicados para comparar la epidemiología de las especies de hongos aquí reportadas.

\section{CONCLUSIONES}

Penicillium olsonii y Alternaria alternata se identificaron como agentes causales de la necrosis floral del mamey $(P$. sapota) en Alpoyeca, Guerrero, México. La intensidad de la enfermedad se correlacionó principalmente con la humedad relativa $(r=0.80)$ y con la velocidad del viento $\leq 5$ $\mathrm{km} \mathrm{h}^{-1}(r=0.75)$. La esporulación de Alternaria alternata y Pestalotiopsis paéoniicola se asoció con la humedad relativa $\left(r_{\text {Alternaria }}=0.75\right.$ y $\left.r_{\text {Pestalotiopsis }}=0.80\right)$. Las epidemias de la necrosis floral del mamey en Alpoyeca, Guerrero se ajustaron al modelo Gompertz (50 \%), logístico (30 \%) y monomolecular $(20 \%)$. 


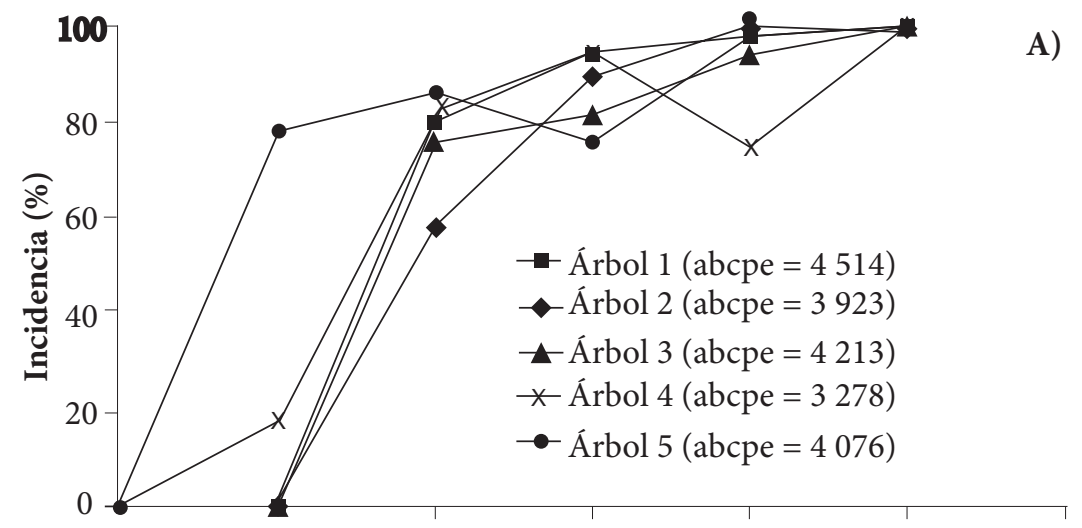

27/05/2005 11/06/2005 25/06/2005 09/07/2005 23/07/2005 06/08/2005 19/08/2005

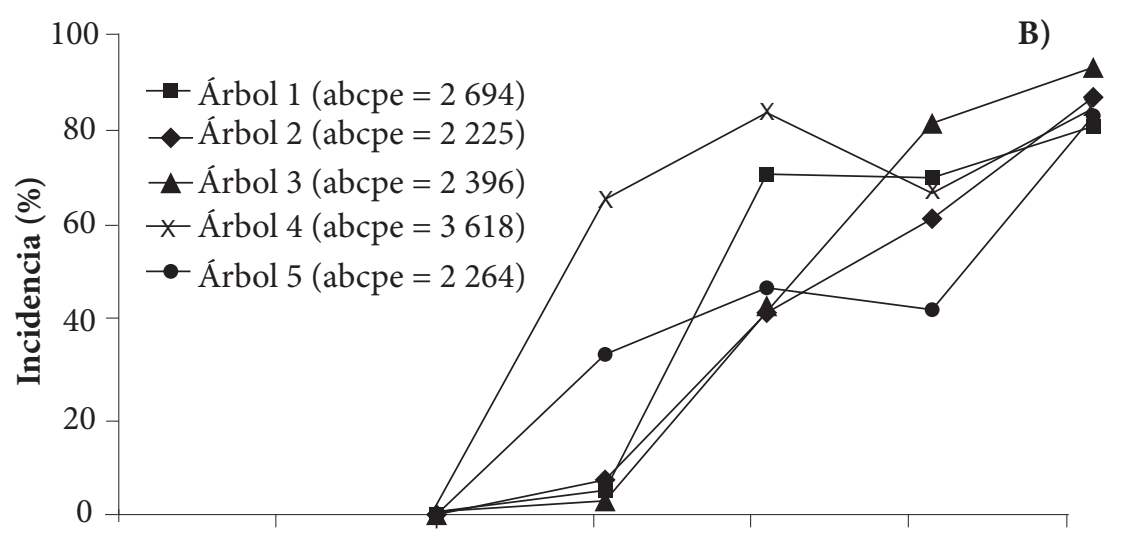

27/05/2005 11/06/2005 25/06/2005 09/07/2005 23/07/2005 06/08/2005 19/08/2005

\section{Fecha de evaluación}

Figura 5. Progreso temporal (\% de incidencia) e intensidad (abcpe) de la enfermedad "necrosis floral" en 10 árboles de mamey ( $P$. sapota) seleccionados en dos huertos comerciales con distinto manejo agronómico, evaluados de mayo a agosto de 2005 en Alpoyeca, Guerrero, México. A) Huerto sin manejo agronómico (HE1). B) Huerto con manejo agronómico (HE2). (abcpe = área bajo la curva del progreso de la enfermedad).

Cuadro 1. Modelos epidemiológicos para caracterizar el progreso temporal de incidencia de necrosis floral (Penicillium olsonii, Alternaria alternata y Pestalotiopsis paeoniicola) en 10 árboles de mamey (Pouteria sapota) de dos huertos experimentales (HE1 y HE2) en Alpoyeca, Guerrero, México, 2005.

\begin{tabular}{|c|c|c|c|c|c|}
\hline Huertos & $\mathrm{R}^{2}$ & Modelo & Ecuación & rho & $\mathrm{k}_{\mathrm{G}}$ \\
\hline \multicolumn{6}{|c|}{ HE1 } \\
\hline Árbol 1 & 0.99 & Monomolecular & $\operatorname{Ln}(1 /(1-y))=0.091 t+0.140$ & 0.046 & 0.182 \\
\hline Árbol 2 & 0.93 & Gompertz & $-\operatorname{Ln}(-\ln (\mathrm{y}))=0.111 \mathrm{t}-1.496$ & & \\
\hline Árbol 3 & 0.92 & Gompertz & $-\operatorname{Ln}(-\ln (y))=0.109 t-1.236$ & & \\
\hline Árbol 4 & 0.70 & Logístico & $\operatorname{Ln}(y /(1-y))=0.121 t-3.650$ & 0.020 & 0.080 \\
\hline Árbol 5 & 0.78 & Gompertz & $-\operatorname{Ln}(-\ln (y))=0.099 t-1.126$ & & \\
\hline \multicolumn{6}{|c|}{ HE2 } \\
\hline Árbol 1 & 0.94 & Logístico & $\operatorname{Ln}(y /(1-y))=0.178 t-5.247$ & 0.030 & 0.118 \\
\hline Árbol 2 & 0.96 & Logístico & $\operatorname{Ln}(y /(1-y))=0.173 t-5.322$ & 0.029 & 0.115 \\
\hline Árbol 3 & 0.98 & Gompertz & $-\operatorname{Ln}(-\ln (y))=0.077 t-1.923$ & & \\
\hline Árbol 4 & 0.75 & Monomolecular & $\operatorname{Ln}(1 /(1-y))=0.036 t+0.252$ & 0.018 & 0.072 \\
\hline Árbol 5 & 0.87 & Gompertz & $-\operatorname{Ln}(-\ln (y))=0.048 \mathrm{t}-1.303$ & & \\
\hline
\end{tabular}




\section{BIBLIOGRAFÍA}

Ahrens U, E Seemüller (1992) Detection de DNA of plant pathogenic mycoplasmalike organisms by polymerase chain reaction that amplifies a sequence of the 16S rRNA gene. Phytopathology 82:828-832.

Azurdia C (2006) Tres Especies de Zapote en América Tropical. Southampon Centre for Underutilised Crops, Universidad de Southampon, Southampon, UK. 216 p.

Balerdi C F, J H Crane, I Maguire (2005) Mamey sapote growing in the Florida Home Landscape. Horticultural Sciences Department, Florida Cooperative Extension Service, Institute of Food and Agricultural Sciences. University of Florida U.S.A. Document FC-30. Disponible en: http://edis.ifas.ufl.edu (Julio 2008).

Barnett L H, B B Hunter (2006) Illustrated Genera of Imperfect Fungi. 4th ed. The American Phytopathological Society. St. Paul, Minnesota, USA. 218 p.

Campbell L C, L V Madden (1990) Introduction to Plant Disease Epidemiology. John Wiley and Sons, Inc. New York, USA. 532 p.

Gadoury M D, W E MacHardy (1983) A 7-day recording volumetric spore trap. Phytopathology 73:1526-1531.

Gómez J R, D Nieto A, D Téliz O, A Mora A, M T Martínez D, M Vargas H (2009) Evaluación de la calidad e incidencia de hongos en frutos refrigerados de zapote mamey (Pouteria sapota (Jacq.) H.E. Moore y Stearn). Agrociencia 43:37-48.

Gottwald T R, M T Trocine, W L Timmer (1997) A computer-controlled environmental chamber for the study of aerial fungal spore release. Phytopathology 87:1078-1084.

Guba E F (1961) Monograph of Monochaetia and Pestalotia. Harvard University Press. Cambridge, Massachusetts, USA. 342 p.

Haldane J B S (1956) The estimation and significance of the logarithm of a ratio of frequencies. Ann. Human Genet. 20:309-314.
Morton J (1987) Sapote: In: Fruits of Warm Climate. J Morton (ed). Miami, Florida, USA. pp:336-346.

Mossler M A, J Crane (2009) Florida crop/pest management profile: mamey sapote and sapodilla. Florida Cooperative Extension Service, Institute of Food and Agricultural Sciences, University of Florida. University of Florida, Gainesville, Fl. U.S.A. Disponible en: http://edis.ifas.ufl.edu (Septiembre 2010).

NCBI, National Center for Biotechnology Information (2008) Blast $\AA$. Disponible en: www.ncbi.nlm.nih.gov/ (Octubre 2008).

Raper K B, C Thom (1949) A Manual of the Penicillia. The Williams \& Wilkins Company. Baltimore, Maryland, USA. 875 p.

Richards F J (1959) A flexible growth function for empirical use. J. Exp. Bot. 10:290-301.

Rotem J (1994) The Genus Alternaria. Biology, Epidemiology, and Pathogenicity. APS Press. St. Paul, Minnesota, USA. 326 p.

Samson R A, E S Hoekstra, J C Frisvad (2004) Introduction to Food- and Airborne Fungi. 7th ed. ASM Press. Utrecht, Netherlands. 389 p.

SIAP, Servicio de Información Agroalimentaria y Pesquera (2011) Agricultura. Disponible en: http://www.siap.gob.mx/index. php?option $=$ com_wrapper\&view $=$ wrapper\&Itemid $=351 \quad(\mathrm{Ju}-$ nio 2012).

Timmer L W, Z Solel, T R Gottwald, M A Ibañez, E S Zitko (1998) Environmental factors affecting production, release, and field populations of conidia of Alternaria alternata, the cause of brown spot of citrus. Phytopathology 88:1218-1223.

Wei J G, T Xu, L D Guo, H X Pan (2005) Endophytic Pestalotiopsis species from Southern China. Mycosystema 24:481-493.

White T J, T D Bruns, S B Lee, J W Taylor (1990) Amplification and direct sequencing of fungal ribosomal RNA genes for phylogenetics. In: PCR Protocols: A Guide to Methods and Applications. M A Innis, D H Gelfand, J J Sninsky, T J White (eds). Academic Press, San Diego, CA, U.S.A. pp:315-322. 